\title{
Heavy Quark Production at the TESLA Collider and its Sensitivity to the Gluon Content in Photon
}

\author{
P. Jankowski and M. Krawczyk \\ Institute of Theoretical Physics, Warsaw University \\ ul. Hoża 69, 00-681 Warsaw, Poland
}

A. De Roeck

CERN, 1211 Geneva 23, Switzerland

\begin{abstract}
Heavy quark production is studied at the high energy linear $e^{+} e^{-}$collider (LC) TESLA both in its nominal and Photon Collider (PC) mode. Leading order cross-sections are calculated for the production of heavy quarks, $e^{+} e^{-} \rightarrow$ $e^{+} e^{-} Q(\bar{Q}) X$, at high transverse momenta. The sensitivity of this process to the gluon content in the photon is studied.
\end{abstract}

An $e^{+} e^{-}$linear collider with a centre of mass system (CMS) energy in the range of 500 to $1000 \mathrm{GeV}$ is the prime candidate for the next high energy accelerator project after the LHC. Recently TESLA [1] has completed a Technical Design Report for such a collider. For the study of a future electron-positron Linear Collider (LC) it is important to examine the physics potential of its main and possible additional options. Such an option is the Photon (or Compton) Collider (PC) in which high energy real photons can be obtained by backscattering photons from a laser beam on the electron or positron beam $[2,3]$. This way an excellent tool for the study of $\gamma \gamma$ collisions at high energies can be provided.

In high energy $e^{+} e^{-}$collisions the hadronic final state is predominantly produced in $\gamma^{\star} \gamma^{\star}$ interactions where the virtual photons $\left(\gamma^{\star}\right)$ are almost on mass shell. Their scattering can be effectively described with the interaction of real photons with energy spectra given by the Weizsäcker-Williams (WW) approximation [4]. Such a spectrum is generally quite soft, as shown in Fig. 1. In contrast, a Photon Collider, based on Compton scattering, provides beams of real photons which are much harder than those from a WW distribution (see Fig. 1). Furthermore these photons can be produced 
in a definite polarization state. While the energy spectrum of the $\mathrm{PC}$ at the high energy end can be well calculated based on a few parameters of the laser and incident electron beam, the lower energy end depends much more on the details of machine and operation. Therefore we limit ourself to study only the high energy peak of $0.6<y=$ $E_{\gamma} / E_{e}<0.83$ [5]. The spectra used in this paper are described in the Appendix.

The main goal of this study is to compare the LC and PC potential for probing the gluon distribution in the photon via heavy quark production. This analysis does not make use of polarization. The measurement of the process $e^{+} e^{-} \rightarrow e^{+} e^{-} Q(\bar{Q}) X$ is an important test of QCD. It is well known that this process is promising for probing the structure of the photon, see e.g. [6, 7] and [8], where a related topic is considered. Heavy quarks can be produced in $\gamma \gamma$ collisions through three mechanisms. Direct (DD) production occurs when both photons couple directly to a $Q \bar{Q}$ pair. In single resolved photoproduction processes (DR) one of photons interacts via its partons with the second photon. When both photons split into a flux of quarks and gluons, the process is labelled a double resolved photon (RR) process.

There are various schemes proposed for calculating cross-sections for processes involving heavy quarks, for the latest overview see ref [9]. Two standard schemes will be used in this analysis ${ }^{1}$. They differ by the number of quark flavours which correspond to partons of the photon (active quarks). In the case of the massive quark scenario, the so called Fixed Flavour Number Scheme (FFNS), the photon "consists" only of light quarks and gluons, which may interact, and massive heavy quarks can be only created in a hard process e.g. via gluon-gluon fusion. The massless quark scheme, Zero-mass Variable Flavour Number Scheme (ZVFNS), considers apart from the gluons and $u, d$ and $s$ quarks also heavy quarks as active quarks, which are all treated massless. All the partonic reactions in both schemes contributing in the LO calculation to heavy quark production are shown in table 1 . Present available data on charm quark production in $\gamma \gamma$ processes is still not precise enough to allow to discriminate between the predictions of these two production schemes. Besides, these theoretical approaches are expected to be in turn appropriate for describing different ranges of the hard scale $(\mu)$ of the process: The massive and massless schemes should be applied below $\left(\mu \leq m_{Q}\right)$ and above $\left(\mu \gg m_{Q}\right)$ the mass scale, respectively. For the charm quark and for transverse momentum $p_{T}$ of the partons larger than e.g. $10 \mathrm{GeV}$, we expect to be closer to the region of applicability of the ZVFNS scheme than the FFNS scheme. For completeness we performed the calculation in both schemes which can be treated as an estimate of the theoretical uncertainty.

We calculate the LO QCD cross-sections for $c$ and $b$ quarks produced with large $p_{T}$ at the $e^{+} e^{-}$LC colliders at energies of 300,500 and $800 \mathrm{GeV}$, respectively, and at the $\mathrm{PC}$ based on the corresponding $\left(e^{+} e^{-}\right) \mathrm{LC}$ with these energies. For comparison we study also the corresponding cross-sections for the LEP collider at $180 \mathrm{GeV}$. To test the sensitivity of the considered processes to the gluonic content of the photon we use two different parton parametrizations for the real photon: GRV [10] and SaS1d [11].

\footnotetext{
${ }^{1}$ a recent attempt to construct a new composite scheme can be found in [9]
} 
We choose the GRV('92) parametrization because in its construction heavy quarks are treated as massless above the heavy quark threshold region $\left(W \gg 2 m_{Q}\right)$, and moreover recent experimental results [12] indicate that GRV gluon distribution is close to the data. The choice of the SaS1d parametrization is motivated by the fact that it provides results in agreement with expectations of Gribov factorization and the QCD sum rule for the structure of the photon [13]. Both these parametrizations are based on QCD fits to photon structure function data measured in $e \gamma$ collisions at $e^{+} e^{-}$experiments $[14,15]$. They have different assumptions for the gluon content, which is only weakly constrained by the data. The GRV and SAS1d distributions both start the evolution from a small scale, $\mu_{0}^{2}=0.25 \mathrm{GeV}^{2}$ and $0.36 \mathrm{GeV}^{2}$, respectively, and consequently both parton densities predict a rise of the gluon density at small $x$. Different treatment of valence quark distributions in the vector mesons leads to a larger gluon component at small- $x_{\gamma}$ of the photon for the GRV set compared to the SAS1d one.

As was mentioned above, in the massive (FFNS) calculations the number of active flavours $\left(N_{f}\right)$ is taken to be 3 . In the massless (ZVFNS) scheme it varies from 3 to 5 depending on the value of the hard (factorization, renormalization) scale $\mu$. The $c(b)$ quarks are included in the computation provided that $\mu>m_{c}\left(m_{b}\right)$ with $m_{c}=1.5 \mathrm{GeV}$ $\left(m_{b}=4.5 \mathrm{GeV}\right)$. When charm quark production is calculated in the ZVFNS scheme the bottom quarks are always excluded, hence $N_{f}=3$ or $N_{f}=4$. Also the QCD energy scale $\Lambda_{Q C D}$, which appears in the one loop formula for the strong coupling constant $\alpha_{s}$, depends on the number of active flavours. We take this scale, denoted as $\Lambda_{Q C D}^{N_{f}}$, to be:

$$
\Lambda_{Q C D}^{3}=232 \quad \Lambda_{Q C D}^{4}=200 \quad \Lambda_{Q C D}^{5}=153 \mathrm{MeV}
$$

as in [10]. The hard scale in the calculation of the cross-section $\mu$ is taken to be the transverse mass of the produced heavy quark $m_{T}=\sqrt{m_{Q}^{2}+p_{T}^{2}}$.

In the calculation we include direct (DD), single (DR) and double resolved (RR) photon processes. We study $\frac{d^{2} \sigma}{d p_{T}^{2} d \eta}$, with $\eta=\frac{1}{2} \ln \frac{E-p_{L}}{E+p_{L}}$ the rapidity of the produced heavy quark and $p_{T}$ its transverse momentum. The results for the charm quark production presented in Figs. 2 and 3 were obtained in the ZVFNS scheme for both types of initial photon spectra. The calculation was performed using the GRV parton parametrization. In Fig. 2 a fixed energy $\sqrt{s}=500 \mathrm{GeV}$ and a $p_{T}=10 \mathrm{GeV}$ for the charm quark was assumed. Both resolved photon contributions, DR and RR, to the process $e^{+} e^{-} \rightarrow e^{+} e^{-} Q(\bar{Q}) X$ are dominated by reactions initiated by gluons, especially for a PC at high $\eta$ values. The dominance of the gluon initiated over the quark initiated processes is larger for the PC spectrum; it is also larger in the FFNS scheme (not shown) compared to the ZVFNS one.

Fig. 3 shows a comparison of the direct (DD), single resolved (DR), double resolved (RR) contributions and their sum for WW and LASER spectra for various CMS energies of the $e^{+} e^{-}$colliders, for charm quarks with a $p_{T}$ of $10 \mathrm{GeV}$. An interesting pattern is observed. In the case of an $e^{+} e^{-} \mathrm{LC}$ with a WW photon spectrum either the direct production of $c$ dominates, or resolved and direct photon contributions are 
found to be of the same size. The DR and RR contributions increase with increasing CMS energy of LC. Nevertheless in the considered range of the LC energies, they do not play a dominant role for heavy quark ${ }^{2}$ production in the $e^{+} e^{-}$mode. The contrary is observed for a PC: charm quark production is always dominated by resolved photon interactions. The direct contribution is limited to high absolute values of the rapidity distribution. For a $p_{T}=10 \mathrm{GeV}$ this rapidity region is outside the range of $|\eta|<2$ which could be covered by a typical detector. Lowering the $p_{T}$ of the observed charm quarks amplifies the contribution of the resolved process to the total cross-section. The direct contribution becomes even less important with increasing CMS energy. Thus the charm production cross-section is much more sensitive to the parton distribution of the photon for a PC compared to a LC. Since for the PC option the resolved photon contributions are dominated by the processes involving gluons (Fig. 2) this offers an excellent tool for measuring the gluonic content of the photon. We note here also that an extention of the experimental reach to observe charm quarks at rapidities beyond $|\eta|=2$ would allow to access a kinematic range where the gluon contribution dominates most strongly over other resolved photon contributions.

For the ZVFNS scheme and the PC option we find that the processes initiated by $c$ quarks constitute about $52-67 \%$ of the total cross-sections presented in Fig. 3, and about $99 \%$ of the contribution which arises due to the processes initiated by quarks (not shown). Since the heavy quark content of the photon is closely related to the gluon density in the photon, the heavy quark production discussed in the paper exhibits also sensitivity to the gluonic density via the heavy quark content of the photon [16]. Since for the LC option the cross-section has a large contribution from the direct process, this effect should be most visible in data from a PC, which are dominated by the resolved photon processes instead.

An important feature of the results for cross-section seen in Fig. 3 is the increase of the resolved photon process contributions, and consequently also an growing sensitivity to gluons, with increasing CMS energy. This arises from the fact that at higher energies we probe regions of small Bjorken- $x_{\gamma}$ values. The minimal $x_{\gamma}$ value that could be reached in $\mathrm{LC}$ for $p_{T}=10 \mathrm{GeV}$ varies from $\sim 0.004$ for $\sqrt{s}=300 \mathrm{GeV}$ to $\sim 0.0006$ for $\sqrt{s}=800 \mathrm{GeV}(\sim 0.01$ in LEP $)$. The detector cut $|\eta|<2$ applied in this analysis increases those values to $\sim 0.006$ and $\sim 0.0018$, respectively. An extra cut on the LASER photon spectrum $(0.6<y<0.83)$ in the PC mode shrinks the $x_{\gamma}$ accesible range even further. The numbers read then: $\sim 0.008$ for $\sqrt{s}=300 \mathrm{GeV}$ and $\sim 0.0023$ for $\sqrt{s}=800 \mathrm{GeV}$. Therefore the RR contribution grows weaker with the CMS energy in the $\mathrm{PC}$ case comparing to the $\mathrm{LC}$ case. For the DR case in the PC mode the $0.6<y<0.83$ cut puts an additional, kinematic constraint. Since one of the photons interacts directly with $E_{\gamma}>0.6 E_{e}$ the energy of the produced heavy quarks must grow with $E_{e}$. If we choose them to have relatively small $p_{T}$ equal to $10 \mathrm{GeV}$ it is more difficult to produce them in the central $\eta$ region where $E \approx p_{T}$. Hence instead of the increase with the energy we see a slight decrease of the DR contribution around $\eta=0$. Due to the same kinematic effect DD events cannot be produced close to $\eta=0$. We

\footnotetext{
${ }^{2} \mathrm{~A}$ similar effect is observed for $b$ quark production (not shown)
} 
note as above that reaching in experiment values of rapidity higher than $|\eta|>2$ would be of high importance. We then could explore kinematic region of strongest resolved contributions to the cross-sections.

The results discussed so far are not significantly affected by the choice of scheme for the heavy quark calculation as is shown in Fig. 4 for the $c$-quark rapidity distribution: for both the FFNS and ZVFNS scheme the cross-section is much larger for the PC than for the LC case.

More details of the comparison of the FFNS and ZVFNS scheme results can be found in Figs. 5-9. The importance of the low $x_{\gamma}$ contributions is demonstrated in Fig. 5, presenting the $\frac{d \sigma}{d x_{\gamma}}$ distributions of the charm production at a $\mathrm{PC}$ with $\sqrt{s_{e e}}=500$ $\mathrm{GeV}$ (for the GRV parton parametrization), on a linear and logarithmic $x_{\gamma}$ scale. The cross-section has been integrated over the region $p_{T}>10 \mathrm{GeV}$ and $|\eta|<2$, realistic for a possible detector at a $\mathrm{PC}$. The direct contribution is included into the bin with the highest $x_{\gamma}$. The double resolved part is counted for twice, once for each $x_{\gamma}$ value. The DR contributions are peaked at small $x_{\gamma}$, while the RR ones have a flatter distribution. The expected differences between both schemes used in the calculation are visible: the ZVFNS scheme gives a larger contribution of DR and RR processes, especially for $x_{\gamma}>0.2$. The maximum in the DR distribution occurs at $x_{\gamma}$ close to 0.01 , independently on the scheme. The RR process have a broad distribution between $x_{\gamma} \sim 0.03-0.05$ and 1.0, and the ZVFNS scheme result is larger by more than one order of magnitude compared to the FFNS one in this whole region.

The sensitivity of the charm quark production process to the gluon distribution is studied further by comparing the predictions obtained using two different parton parametrizations for the photon. In Fig. 6(7) the ratio of the relative difference of the cross-sections $\frac{d^{2} \sigma}{d p_{T}^{2} d \eta}$ is presented, obtained using the GRV and SaS1d parton parametrizations in the ZVFNS (FFNS) scheme. As expected the cross-section obtained using the PC photon spectrum (LASER) leads to a larger sensitivity than the WW spectrum for a given energy of the $e^{+} e^{-}$collider: the difference between results of these two parametrizations shown is $5-20 \%$ for a WW and $30-50 \%$ for LASER photon spectrum. Hence a PC collider has a larger sensitivity for testing the photon structure and especially its gluon density. It is worth mention that these results indicate however that an initial study of the partonic structure of real photon can be already made with an $e^{+} e^{-}$LC itself.

Next we show some detailed characteristics of charm quark production at a PC. Fig. 8 and 9 show the relative difference of $x_{\gamma}$ distribution calculated with the GRV and SaS1d parametrizations, for the $\frac{d \sigma}{d x_{\gamma}}$ cross-sections introduced before, for $|\eta|<2$. Results for $p_{T}>10 \mathrm{GeV}$ are shown in Fig. 8 (for both linear (A) and logarithmic (B) scale) and for $p_{T}>5 \mathrm{GeV}$ in Fig. 9 (linear scale only).

Table 2 shows in detail how the gluon initiated resolved processes dominate the charm quark production. For the case of $\sqrt{s}=800 \mathrm{GeV}$ they contribute $76 \%$ and $98 \%$ 
of the integrated cross-section calculated in the FFNS and ZVFNS scheme respectively. Further details on contributions of the gluon and charm quark initiated processes can be found in Table 3 .

Up to now we presented only the results for $c$ quark production. The corresponding $b$ quark production has all the features listed above though the difference between a PC and a LC was found to be smaller. All calculated cross-sections for bottom production are found to be even more sensitive to the shape of gluonic content of the photon than the corresponding ones for charm quark production, but the cross-sections are smaller.

Next we calculate the number of events with heavy quarks, $c$ and $b$, assuming an $e^{+} e^{-}$integrated luminosity of $300 \mathrm{fb}^{-1}$, which could be achieved at a high luminosity $\mathrm{LC}$ with one year of running. The corresponding luminosity of the $\mathrm{PC}$ for the region $0.6<y<0.83$ is about a factor 3 to 4 lower [17]. In the FFNS scheme the event rates for charm quark production at a LC for the different CMS energies 300-800 GeV amount to $1-4 \cdot 10^{6}$ events for $p_{T}>10 \mathrm{GeV}$; the corresponding numbers at a PC are $9-25 \cdot 10^{6}$ events. Hence these calculations predict a high number of the heavy quarks, $c$ and $b$, produced at the considered CMS energies of the $e^{+} e^{-}$collider of 300, 500 and $800 \mathrm{GeV}$ for both the $\mathrm{LC}$ and $\mathrm{PC}$ options.

In practice, charm quarks with a $p_{T}>5 \mathrm{GeV}$ produced e.g. via $D^{*}$ decays can be detected in a generic LC detector without dedicated detectors in the rapidity range of $|\eta|<1.5-2$. This restricts the lowest reachable $x_{\gamma}$ values to 0.0011 and 0.0023 for a minimum $p_{T}$ of 5 and $10 \mathrm{GeV}$, respectively, for a $\mathrm{PC}$ collider at $800 \mathrm{GeV}$. The cross-sections for $c$ and $b$ production in this phase space region are presented in Table 4. The corresponding event rates (for FFNS scheme leading to smaller rates) are given in Table 5. It shows that the event rate at a PC is still larger than the one at a LC, but the difference is reduced to a factor 2 to 4 . Hence some of the advantage of the $\mathrm{PC}$ over the LC is lost due to the charm quark production at large $\eta$ in case of the PC (see Fig. 4), which will go undetected with the presently planned detectors.

To measure the charm content of the photon e.g. $D^{*}$ mesons can be selected to reconstruct the kinematics of the scattering process. The efficiency including fragmentation fraction and branching ratios, is typically around a few times $10^{-3}$ [18]. Hence the number of reconstructed events per year with charm quark with $p_{T}>10 \mathrm{GeV}$ will be approximately a few thousand for the LC and about ten thousand events for the $\mathrm{PC}$. The numbers are about 5 times larger for a reduced cut of $p_{T}>5 \mathrm{GeV}$. These data will clearly allow for precision measurements of charm quark production and further on the gluon distribution in the photon. The statistical precision of the measurements of $\frac{d^{2} \sigma}{d p_{T}^{2} d \eta}$ with e.g. ten bins will be approximately $5 \%$ at the $\mathrm{LC}$ and a few $\%$ at the $\mathrm{PC}$. The event rates in the ZVFNS scheme can be larger by a factor 3, compared to the FFNS scheme.

In conclusion, the calculated cross-sections for heavy quarks ( $c$ and $b$ ) production in two photon collisions show a higher sensitivity to the parton distributions of the photon in case of a Photon Collider compared to a $e^{+} e^{-}$Linear Collider. This does not depend on the particular scheme used to calculate the heavy quark cross-sections. 
Since the resolved photon contribution is to a large extent dominated by gluon induced processes, especially for a high energy PC, we conclude that heavy quark production provides indeed a sensitive probe of the gluon content of the photon. Combining the above features with the larger cross-sections achieved at energies of the $e^{+} e^{-}$collisions at a PC favours this option for future photon structure research. A high luminosity $e^{+} e^{-}$collider which drives the PC collider will however be essential.

\section{Appendix}

The following formula of the Equivalent Photon Approximation is used:

$$
\begin{array}{r}
f_{\gamma}(y)=\frac{\alpha}{2 \pi}\left(\frac{\left(1+(1-y)^{2}\right)}{y} \log \left(\frac{Q_{\max }^{2}}{Q_{\min }^{2}}\right)-\frac{2(1-y)}{y}\left(1-\frac{Q_{\min }^{2}}{Q_{\max }^{2}}\right)\right) \\
Q_{\min }^{2}=\frac{m_{e}^{2} y^{2}}{1-y} \\
Q_{\max }^{2}=1 \mathrm{GeV} V^{2}
\end{array}
$$

where $y=\frac{E_{\gamma}}{E_{e}}$ and $m_{e}$ is the mass of the electron.

In case of the PC mode we use the original energy spectrum of unpolarized photons $[2]:$

$$
\begin{array}{r}
f_{\gamma}(y)=\frac{1}{\sigma_{c}^{n p}}\left[\frac{1}{1-y}+1-y-4 r(1-r)\right], \\
\sigma_{c}^{n p}=\left(1-\frac{4}{\kappa}-\frac{8}{\kappa^{2}}\right) \ln (\kappa+1)+\frac{1}{2}+\frac{8}{\kappa}-\frac{1}{2(\kappa+1)^{2}}, \\
r=\frac{y}{\kappa(1-y)},
\end{array}
$$

where $\kappa$ is a parameter giving the restriction of $y$ value: $y<\frac{\kappa}{1+\kappa}$. It is argued [3] that the optimal value of $\kappa$ is 4.83 , which gives a cut of $y, y_{\max }=0.83$. We have chosen these values for this analysis. Because the part of the spectrum with $y<0.6$ is very sensitive to the technical parameters of the PC such as the size of the beam we used only its high energy peak of $0.6<y<0.83$ [5].

\section{Acknowledgment}

M.K. has been partly supported by the Polish State Committee for Scientific Research 5 P03B12120 (2001) and European Commission 5th framework contract HPRNCT-2000-00149 


\section{References}

[1] TESLA Technical Design Report, DESY 2001-011, ECFA 2001-209, TESLA Report 2001-23, TESLA-FEL 2001-05, March 2001.

[2] I.F.Ginzburg, G.L.Kotkin, V.G.Serbo and V.I.Telnov, Nucl. Instr. and Meth. 205 (1983) 47; I.F.Ginzburg, G.L.Kotkin, S.L.Panfil, V.G.Serbo and V.I.Telnov, Nucl. Instr. and Meth. 219 (1984) 5.

[3] V.I.Telnov, Nucl. Instr. and Meth. A294 (1990) 72.

[4] C. F. Weizsäcker, Z. Phys. 88 (1934) 612; E.J. Williams, Phys. Rev. 45 (1934)729.

[5] B.Badelek et al., TESLA Technical Design Report, Part 6, Chapter 1, The Photon Collider at TESLA, DESY 2001-011, ECFA 2001-209, TESLA Report 2001-23, TESLA-FEL 2001-05, March 2001. e-Print: hep-ex/0108012

[6] M.Drees,M.Krämer,J.Zunft and P.M.Zerwas, Phys. Lett. B306 (1993) 371.

[7] M.Cacciari, M.Greco, B.A.Kniehl, M.Krämer, G.Kramer and M.Spira, Nucl. Phys. B466, (1996) 173.

[8] M. Doncheski, S. Godfrey and K.A. Peterson, Phys. Rev. D55, (1997) 183.

[9] W.Tung, S.Kretzer and C.Schmidt, To appear in the proceedings of New Trends in HERA Physics 2001, Ringberg Castle, Tegernsee, Germany, 17-22 Jun 2001. hep-ph/0110247

[10] M. Glück, E. Reya and A. Vogt, Phys. Rev. D46, (1992) 1973.

[11] G.A. Schuler and T. Sjöstrand, Z. Phys. C68, 607 (1995); Phys. Lett. B376, (1996) 193.

[12] C. Adloff et al., Euro. Phys. J. C10, (1999) 363

[13] V.N. Gribov and L.Ya. Pomeranchuk, Phys. Rev. Lett. 8, (1962) 343; L.L. Frankfurt and E.G. Gurvich, Phys. Lett. B386, (1996) 379; H. Abramowicz, E. Gurvich and A. Levy, Phys. Lett. B420, (1998) 104

[14] R. Nisius, Phys. Rep. 332 (2000) 166

[15] M. Krawczyk, A. Zembrzuski, M. Staszel, Phys. Rep. 345 (2001) 268.

[16] M. Krämer, private communication.

[17] E. Boos et al., Nucl. Instr. and Meth. A472, (2001) 100

[18] OPAL Collaboration, G. Abbiendi et al., Eur. Phys. J. C16, (2000) 579 


\begin{tabular}{|c|c|c|c|}
\hline & DD & DR & RR \\
\hline FFNS & $\gamma+\gamma \rightarrow Q+Q$ & $g+\gamma \rightarrow Q+Q$ & $g+g \rightarrow Q+\bar{Q}$ \\
& & & $q+\bar{q} \rightarrow Q+\bar{Q}$ \\
\hline ZVFNS & $\gamma+\gamma \rightarrow Q+Q$ & $g+\gamma \rightarrow Q+Q$ & $g+g \rightarrow Q+Q$ \\
& & $Q(\bar{Q})+\gamma \rightarrow Q(\bar{Q})+g$ & $q+\bar{q} \rightarrow Q+\bar{Q}$ \\
& & & $Q+\bar{Q} \rightarrow Q+\bar{Q}$ \\
& & & $Q+Q(\bar{Q}+\bar{Q}) \rightarrow Q+Q(\bar{Q}+\bar{Q})$ \\
& & & $Q(\bar{Q})+q(\bar{q}) \rightarrow Q(\bar{Q})+q(\bar{q})$ \\
& & & $Q(\bar{Q})+g \rightarrow Q(\bar{Q})+g$ \\
\hline
\end{tabular}

Table 1: Parton reactions contributing to heavy quark production in $\gamma \gamma$ collision in the massive (FFNS) and massless (ZVFNS) schemes.

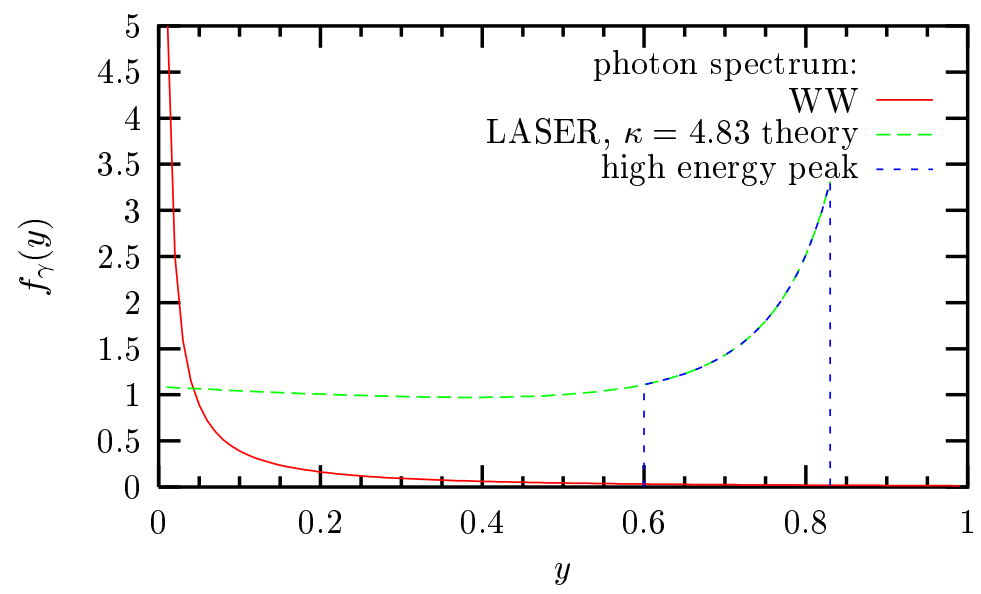

Figure 1: The photon spectra $f_{\gamma}(y)$ as a function of the energy fraction of the photon. A comparison is shown of the spectrum of photons radiated by the initial electron according to the Weizsäcker-Williams (WW) approximation with the spectrum of (unpolarized) photons produced in the backscattering Compton process on the laser beam (LASER) for $\kappa=4.83$, see Appendix for definitions. For the LASER case the high energy peak $y>0.6$ is indicated. 

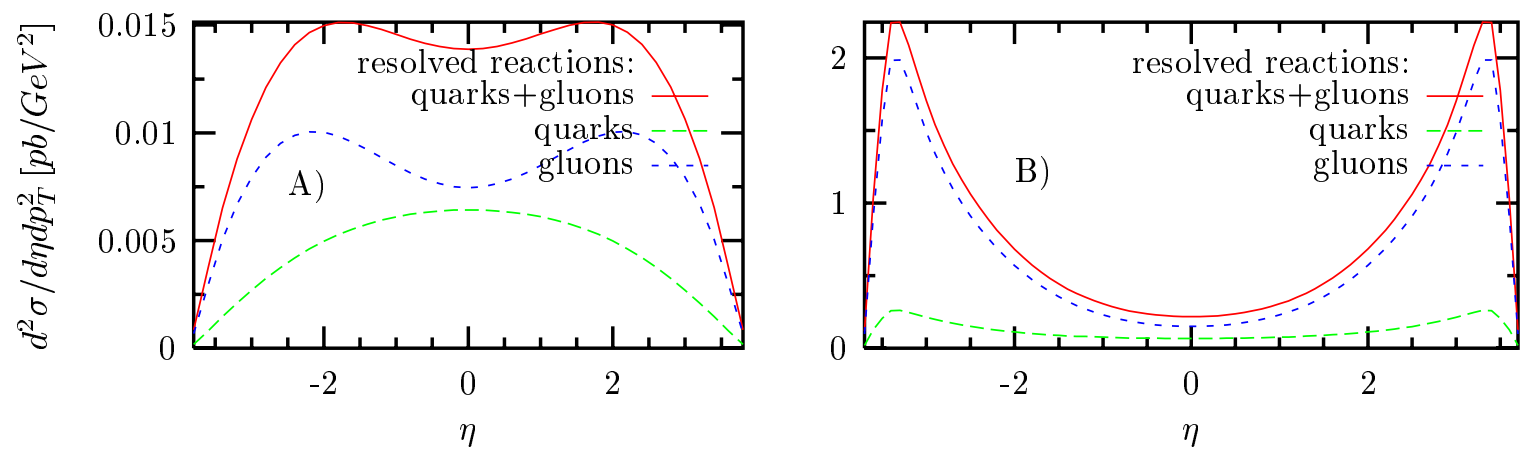

Figure 2: Comparison of parton processes which involve gluons with other partonic contributions to the resolved ( $\mathrm{DR}+\mathrm{RR}$ ) part of the cross-section $\frac{d^{2} \sigma}{d p_{T}^{2} d \eta} e^{+} e^{-} \rightarrow e^{+} e^{-} c / \bar{c} X$ for $\sqrt{s}=500 \mathrm{GeV}$. Results are obtained for charm quarks with transverse momentum $p_{T}=10 \mathrm{GeV}$ as a function of rapidity $\eta$ (in the $e^{+} e^{-}$CM frame) in the ZVFNS scheme with the GRV parton parametrization using: A) the WW spectrum, B) the LASER spectrum. 

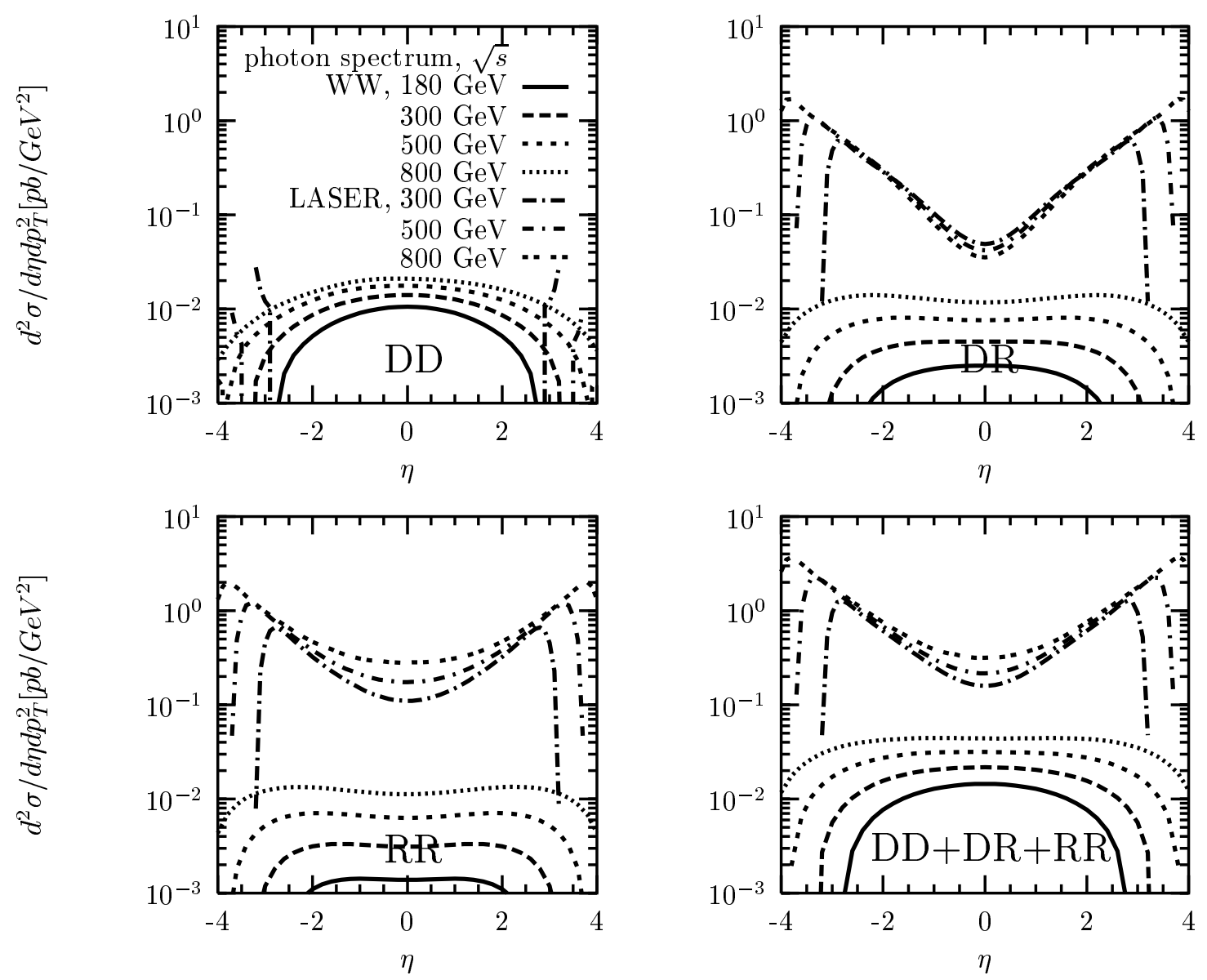

Figure 3: The cross-sections $\frac{d^{2} \sigma}{d p_{T}^{2} d \eta}\left(e^{+} e^{-} \rightarrow e^{+} e^{-} c / \bar{c} X\right)$ at various LC energies as a function of the $c / \bar{c}$ rapidity $\eta$ in the $e^{+} e^{-} \mathrm{CM}$ frame at $p_{T}=10 \mathrm{GeV}$ presented for two types of photon spectra (WW and LASER). Separately the DD, DR, RR and their sum are shown. For a comparison also estimates for LEP $2(\mathrm{WW}, \sqrt{s}=180 \mathrm{GeV})$ are given. The GRV parton parametrization and the ZVFNS scheme are used. 


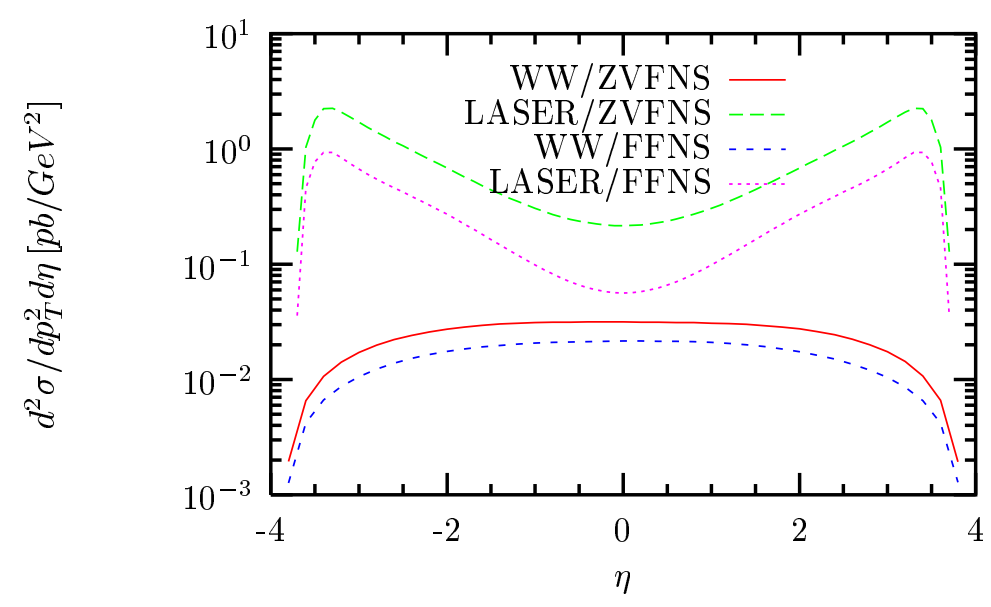

Figure 4: The cross-section $\frac{d^{2} \sigma}{d p_{T}^{2} d \eta}\left(e^{+} e^{-} \rightarrow e^{+} e^{-} c / \bar{c} X\right)$ at $\sqrt{s}=500 \mathrm{GeV}$ as a function of $\eta$ for $p_{T}=10 \mathrm{GeV}$. In the calculation the GRV parton parametrization is used. A comparison is shown between ZVFNS and FFNS schemes, and between the WW and LASER photon spectra.
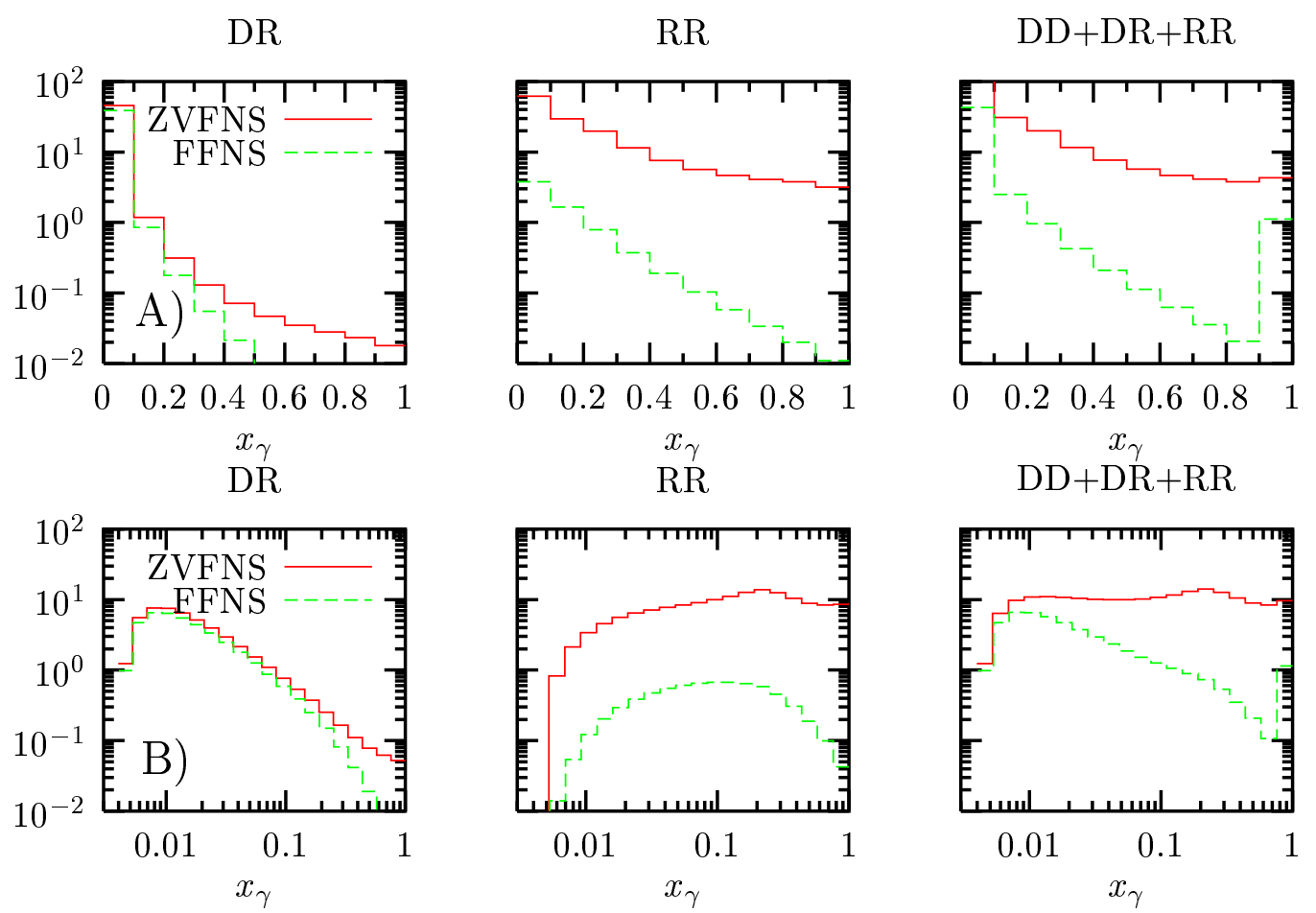

Figure 5: The cross-section $\frac{d \sigma}{d x_{\gamma}}\left(e^{+} e^{-} \rightarrow e^{+} e^{-} c / \bar{c} X\right)$ as a function of $x_{\gamma}$ for $\sqrt{s}=500$ $\mathrm{GeV}$, integrated over $p_{T}>10 \mathrm{GeV}$ and $|\eta|<2$ for two types of schemes, ZVFNS and FFNS. The LASER spectrum and the GRV parton parametrization are used; A) linear, B) logarithmic $x_{\gamma}$ scale. 


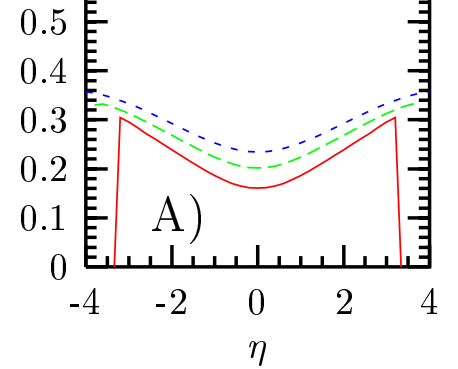

DR

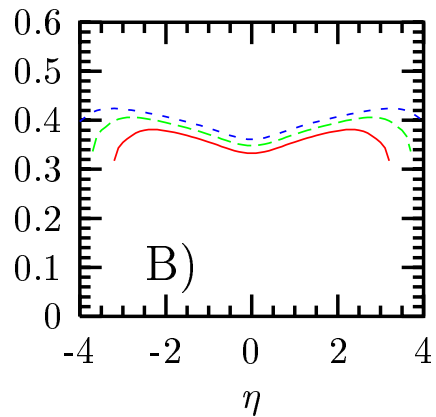

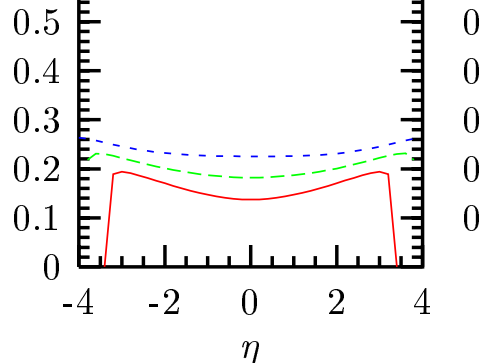

$\mathrm{RR}$

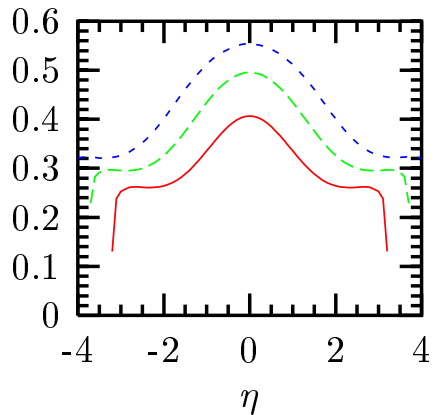

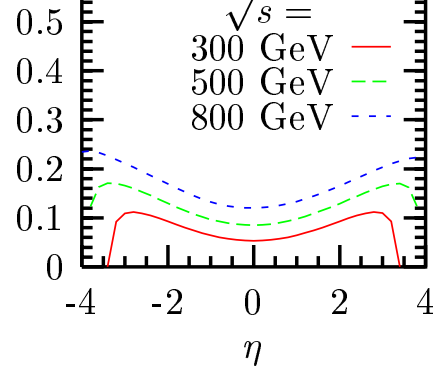

$\mathrm{DD}+\mathrm{DR}+\mathrm{RR}$

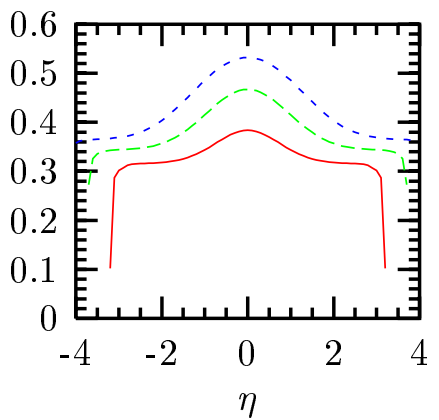

Figure 6: The ratio $\frac{G R V-S A S 1 d}{G R V}$ of the cross-section $\frac{d^{2} \sigma}{d p_{T}^{2} d \eta}\left(e^{+} e^{-} \rightarrow e^{+} e^{-} c / \bar{c} X\right)$ as a function of $\eta$ for various LC energies and for $p_{T}=10 \mathrm{GeV}$. Results are obtained in the ZVFNS scheme for two photon spectra: A) WW, B) LASER.

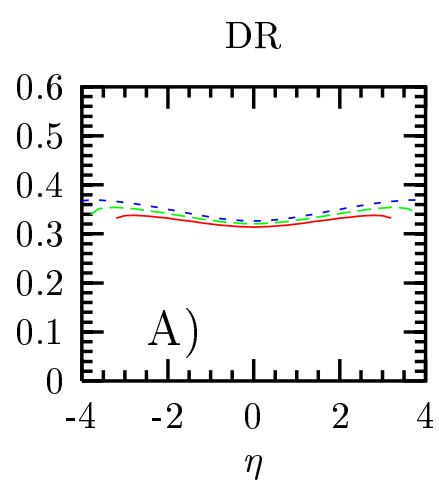

DR

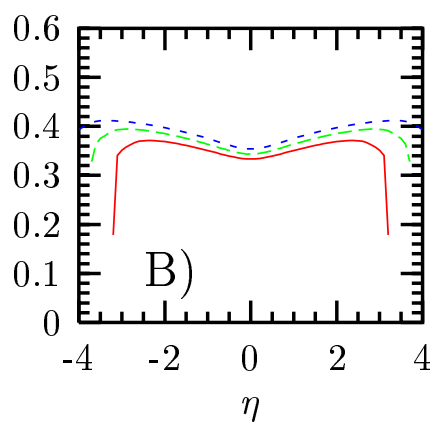

$\mathrm{RR}$

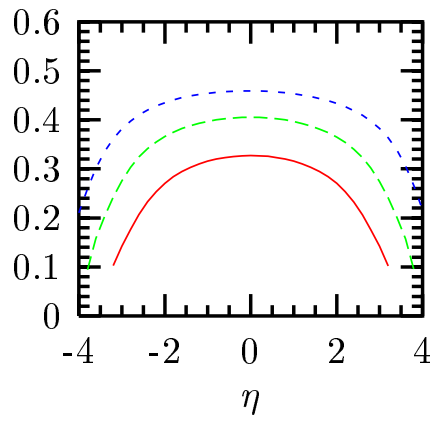

$\mathrm{RR}$

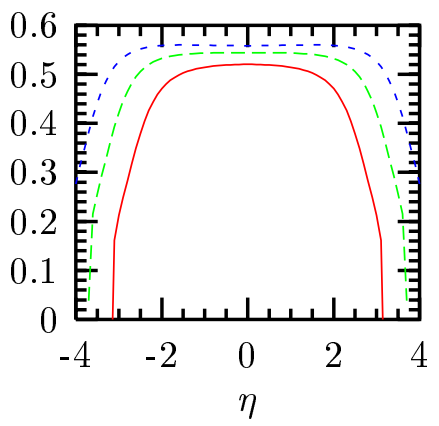

$\mathrm{DD}+\mathrm{DR}+\mathrm{RR}$

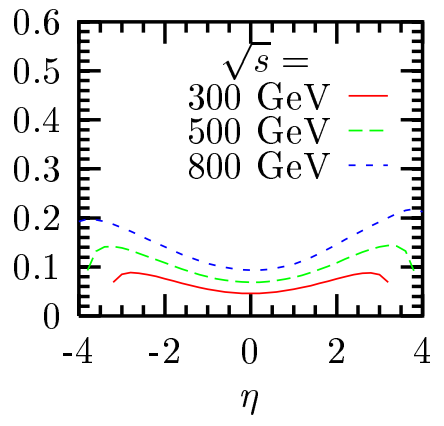

$\mathrm{DD}+\mathrm{DR}+\mathrm{RR}$

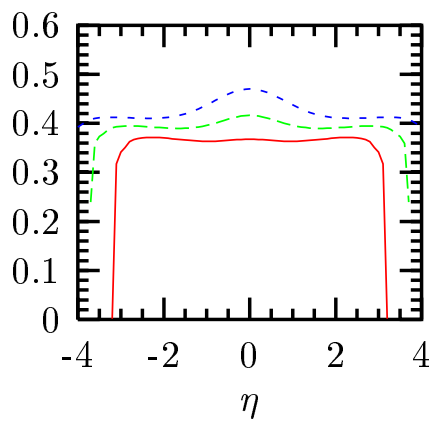

Figure 7: As in Fig. 6, for the FFNS scheme. 
DR

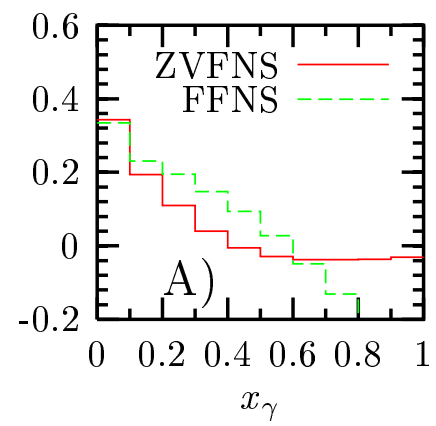

DR

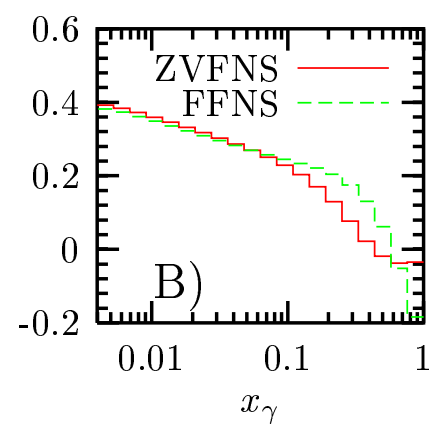

RR

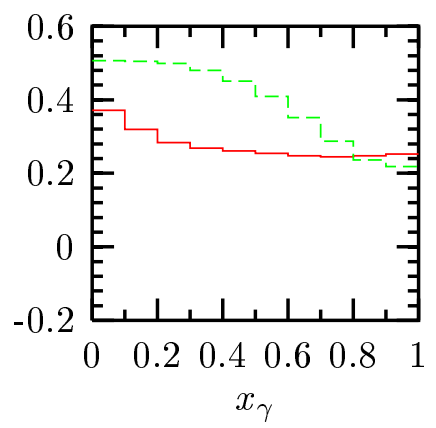

$\mathrm{RR}$

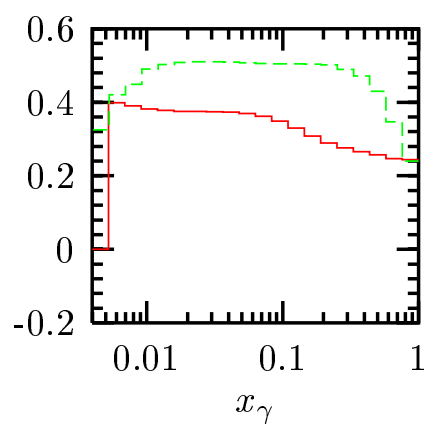

$\mathrm{DD}+\mathrm{DR}+\mathrm{RR}$

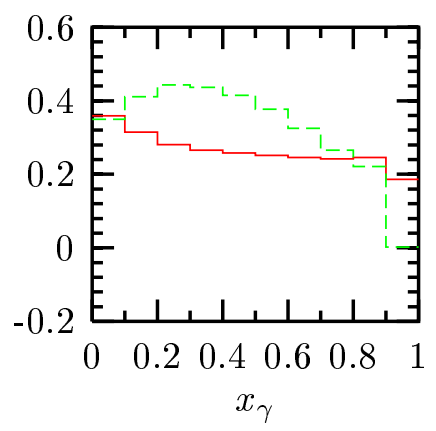

$\mathrm{DD}+\mathrm{DR}+\mathrm{RR}$

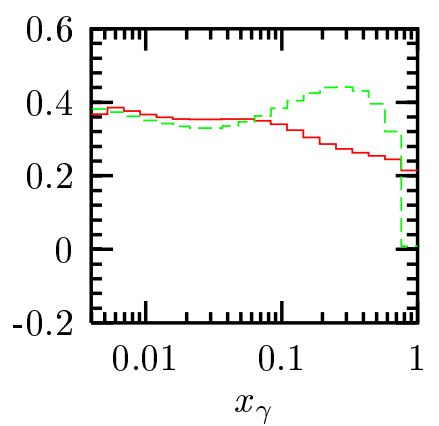

Figure 8: The ratio $\frac{G R V-S A S 1 d}{G R V}$ of the cross-section $\frac{d \sigma}{d x_{\gamma}}\left(e^{+} e^{-} \rightarrow e^{+} e^{-} c / \bar{c} X\right)$ as a function of $x_{\gamma}$ at $\sqrt{s}=500 \mathrm{GeV}$ for $p_{T}>10 \mathrm{GeV}$ and $|\eta|<2$. A comparison is shown of the two schemes for a LASER spectrum on A) linear and B) logarithmic $x_{\gamma}$ scale.

DR

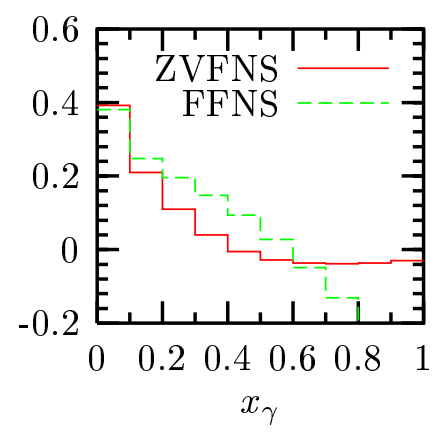

RR

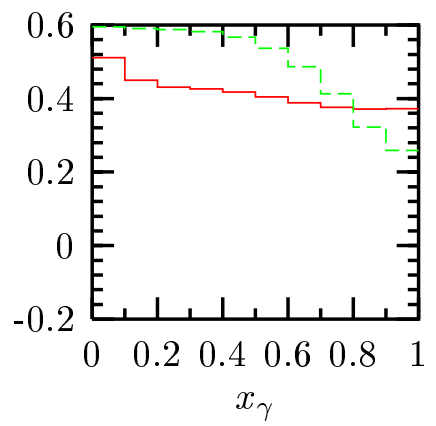

$\mathrm{DD}+\mathrm{DR}+\mathrm{RR}$

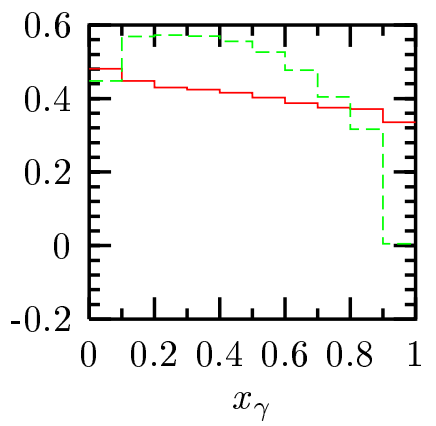

Figure 9: As in Fig. 8, for $p_{T}>5 \mathrm{GeV}$ and linear scale only. 


\begin{tabular}{|c|c|c|c|c|c|c|c|c|}
\hline$p_{T}>10 \mathrm{GeV}$ & DD & \multicolumn{2}{|c|}{ DR } & \multicolumn{5}{c|}{$\mathrm{RR}$} \\
\hline & $\gamma+\gamma$ & $g+\gamma$ & $Q+\gamma$ & $g+g$ & $q+\bar{q}$ & $Q+Q, Q+Q$ & $Q+q$ & $Q(\bar{Q})+g$ \\
\hline$\sqrt{s}=300 \mathrm{GeV}$ & 3.1 & 40 & 5.6 & 0.34 & 5.5 & 1.3 & 17 & 33 \\
\hline$\sqrt{s}=500 \mathrm{GeV}$ & 1.1 & 43 & 4.6 & 0.37 & 6.3 & 3.5 & 20 & 46 \\
\hline$\sqrt{s}=800 \mathrm{GeV}$ & 0.43 & 42 & 3.8 & 0.41 & 7.5 & 7.9 & 24 & 62 \\
\hline
\end{tabular}

Table 2: Cross-sections (in $p b$ ) for charm quark production with $p_{T}>10 \mathrm{GeV}$ and $|\eta|<2(0.6<y<0.83)$, calculated in the massless ZVFNS scheme with the GRV parton parametrization.

\begin{tabular}{|c|c|c|c|c|}
\hline$p_{T}>10 \mathrm{GeV}$ & \multicolumn{3}{|c|}{ ZVFNS } & FFNS \\
\hline & $\sigma(g) / \sigma$ & $\sigma(c) / \sigma$ & $\sigma(g+c) / \sigma$ & $\sigma(g) / \sigma$ \\
\hline$\sqrt{s}=300 \mathrm{GeV}$ & 0.70 & 0.58 & 0.97 & 0.92 \\
\hline$\sqrt{s}=500 \mathrm{GeV}$ & 0.74 & 0.61 & 0.99 & 0.97 \\
\hline$\sqrt{s}=800 \mathrm{GeV}$ & 0.76 & 0.66 & 0.99 & 0.98 \\
\hline
\end{tabular}

Table 3: An estimate of the partonic contributions to the charm quark production with $p_{T}>10 \mathrm{GeV}$ and $|\eta|<2(0.6<y<0.83)$, calculated in both the massless ZVFNS and massive FFNS schemes with the GRV parton parametrization. The sum of the contributions which involve gluons $(\sigma(g))$, charm quarks $(\sigma(c))$ or both $(\sigma(g+c))$ in the initial state are compared to the total cross-section $\sigma$. 


\begin{tabular}{|c|c|c|c|c|c|c|}
\hline & \multicolumn{3}{|c|}{$p_{T}>10 \mathrm{GeV}$} & \multicolumn{2}{c|}{$p_{T}>5 \mathrm{GeV}$} \\
\hline & \multicolumn{2}{|c|}{$c / \bar{c}$} & \multicolumn{2}{c|}{$b / b$} & \multicolumn{2}{c|}{$c / \bar{c}$} \\
\hline & LC & PC & LC & PC & LC & PC \\
\hline$\sqrt{s}=300 \mathrm{GeV}$ & $4.0(5.4)$ & $42(110)$ & $0.27(0.22)$ & $8.4(23)$ & $25(39)$ & $170(490)$ \\
\hline$\sqrt{s}=500 \mathrm{GeV}$ & $6.0(8.6)$ & $45(120)$ & $0.46(0.94)$ & $10(32)$ & $35(58)$ & $180(600)$ \\
\hline$\sqrt{s}=800 \mathrm{GeV}$ & $8.2(13)$ & $47(150)$ & $0.73(1.6)$ & $13(47)$ & $47(83)$ & $200(770)$ \\
\hline
\end{tabular}

Table 4: Cross-sections (in $p b$ ) for heavy quark production with $|\eta|<2$, at a LC and a $\mathrm{PC}(0.6<y<0.83)$, calculated in the massive FFNS (ZVFNS) scheme with the GRV parton parametrization.

\begin{tabular}{|l|c|c|c|c|c|c|}
\hline & \multicolumn{3}{|c|}{$p_{T}>10 \mathrm{GeV}$} & \multicolumn{2}{c|}{$p_{T}>5 \mathrm{GeV}$} \\
\hline & \multicolumn{2}{|c|}{$c / \bar{c}$} & \multicolumn{2}{c|}{$b / b$} & \multicolumn{2}{c|}{$c / \bar{c}$} \\
\hline & LC & PC & LC & PC & LC & PC \\
\hline$\sqrt{s}=300 \mathrm{GeV}$ & 1.2 & 3.9 & 0.08 & 0.80 & 7.5 & 16 \\
\hline$\sqrt{s}=500 \mathrm{GeV}$ & 1.8 & 4.1 & 0.14 & 0.97 & 11 & 17 \\
\hline$\sqrt{s}=800 \mathrm{GeV}$ & 2.5 & 4.4 & 0.22 & 1.2 & 14 & 19 \\
\hline
\end{tabular}

Table 5: Event rates (in units of $\left.10^{6}\right)$ for heavy quark production with $|\eta|<2$ at a $\mathrm{LC}$ and a PC $(0.6<y<0.83)$, calculated in the massive FFNS scheme with the GRV parton parametrization. The $e^{+} e^{-}$LC luminosity was assumed to be $300 \mathrm{fb}^{-1}$. 


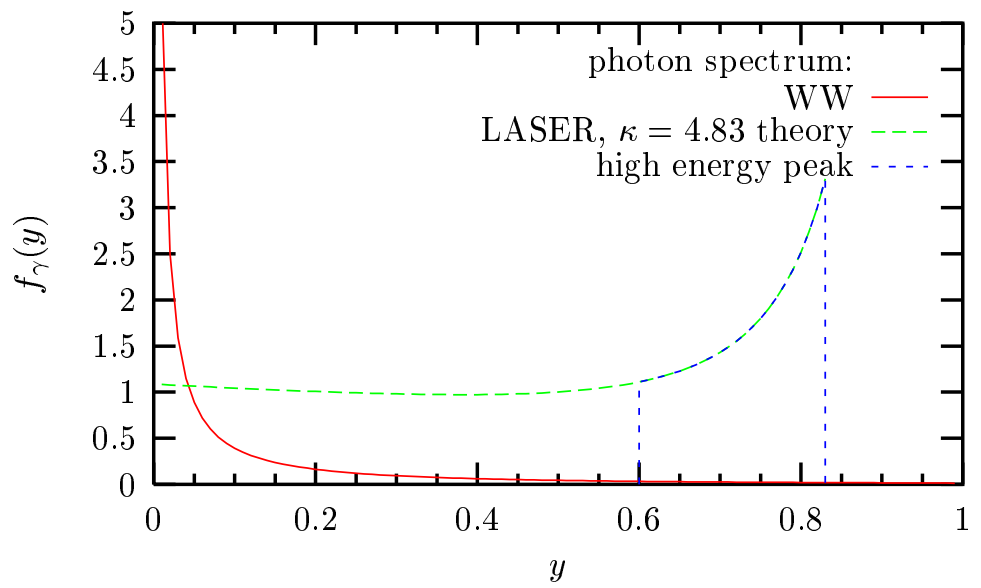



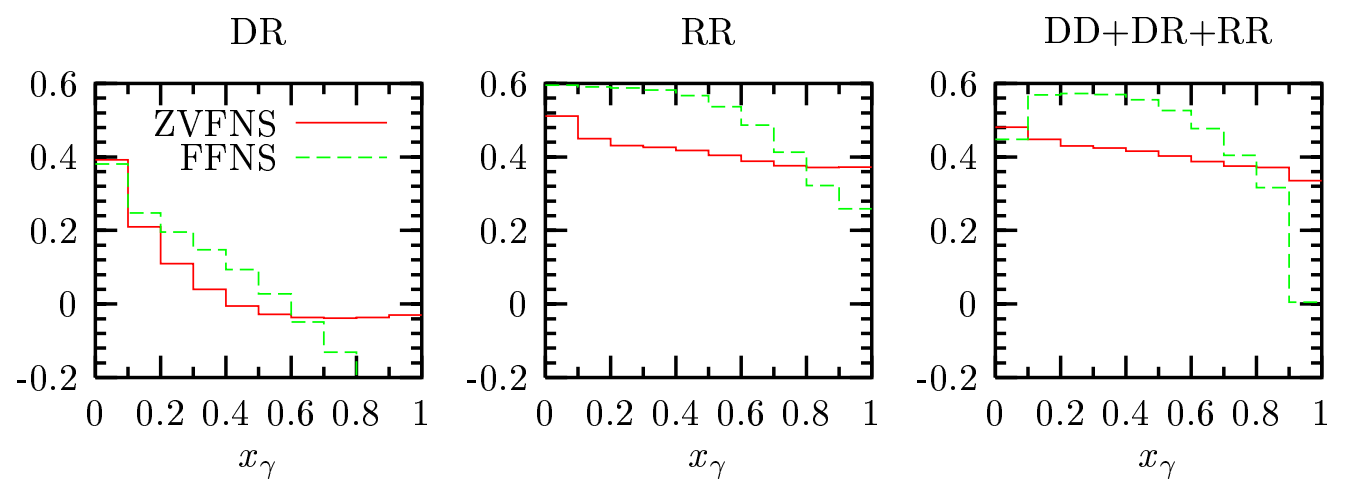

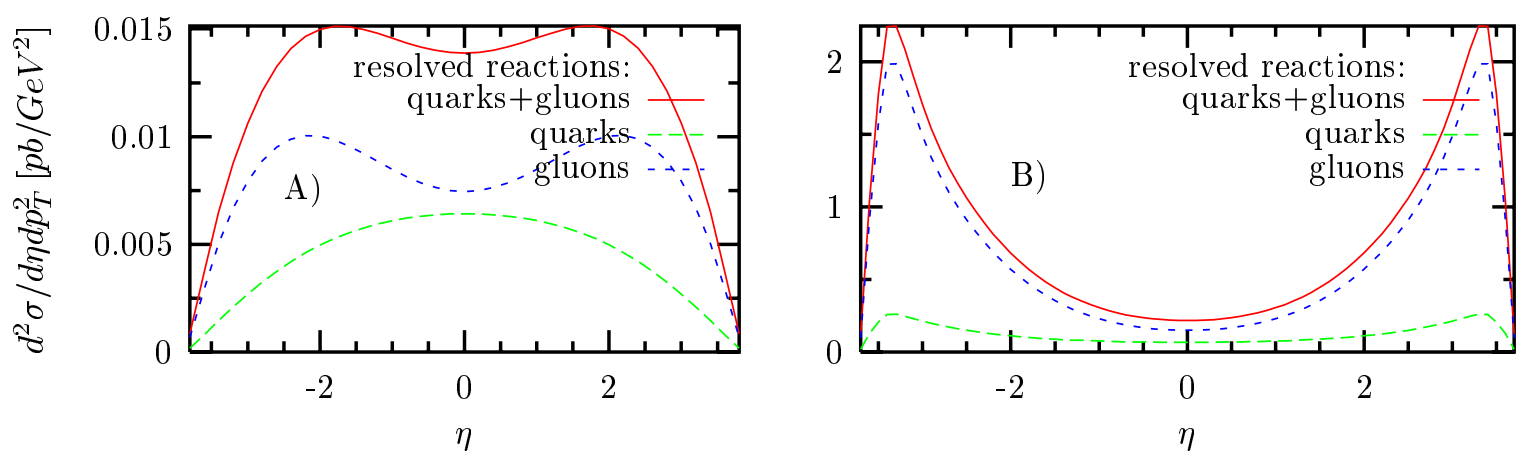

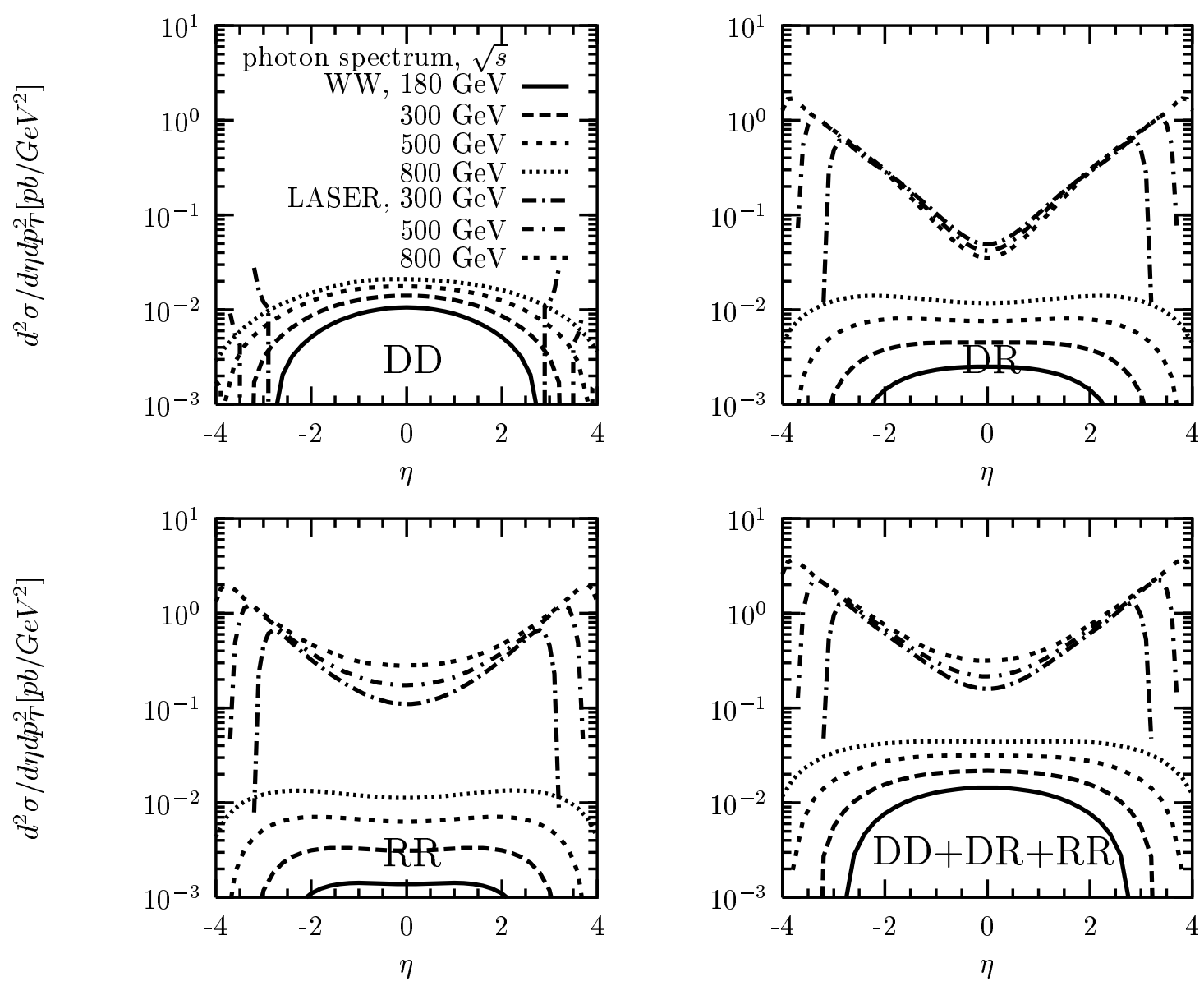


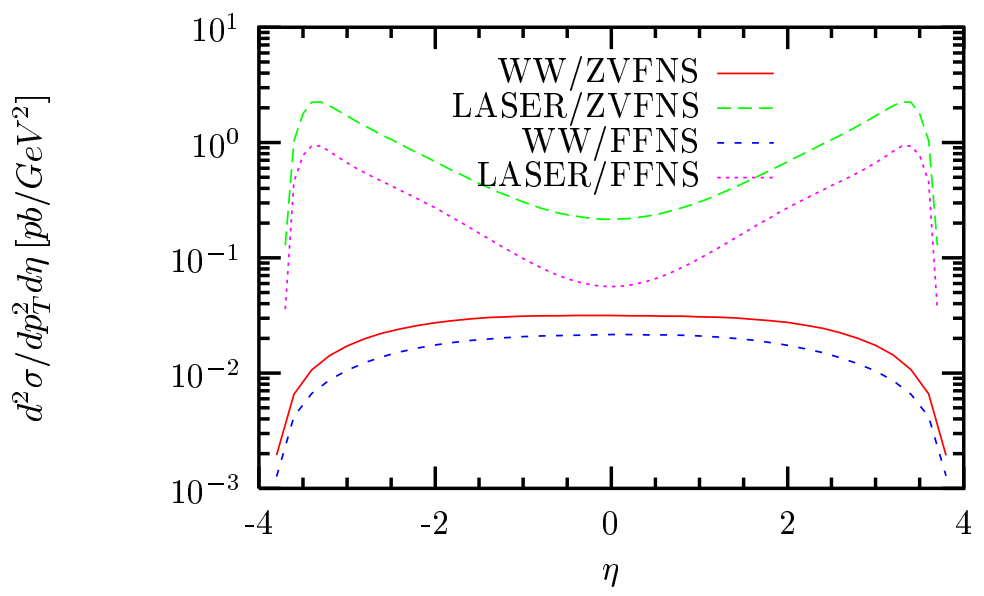




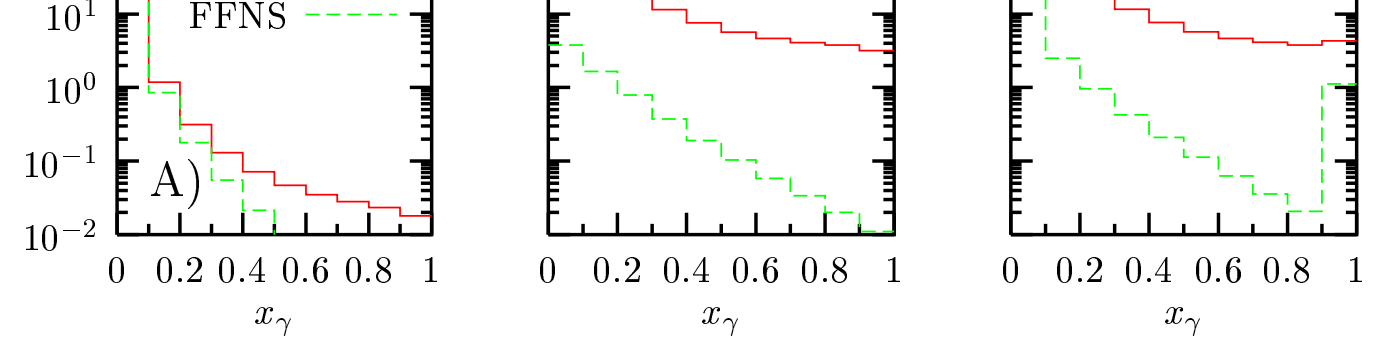



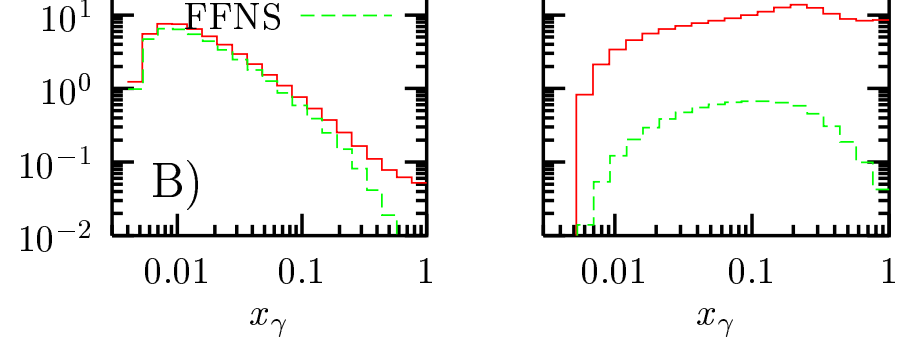

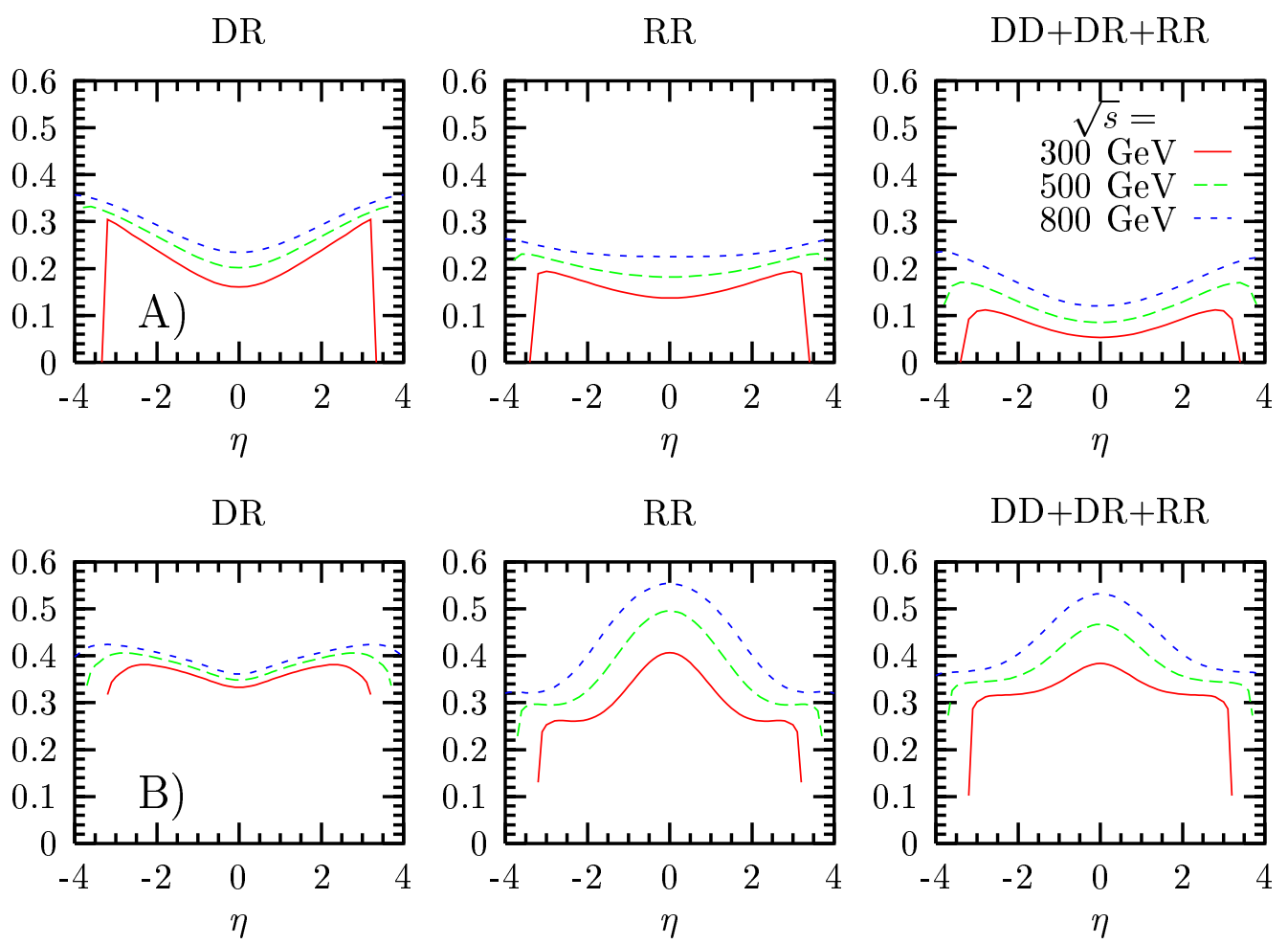
DR

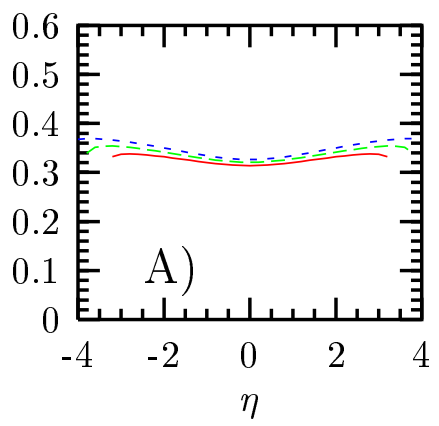

DR

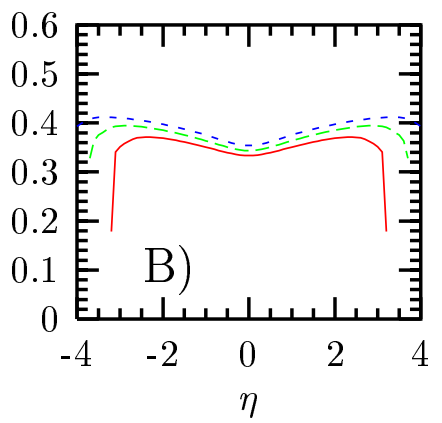

RR

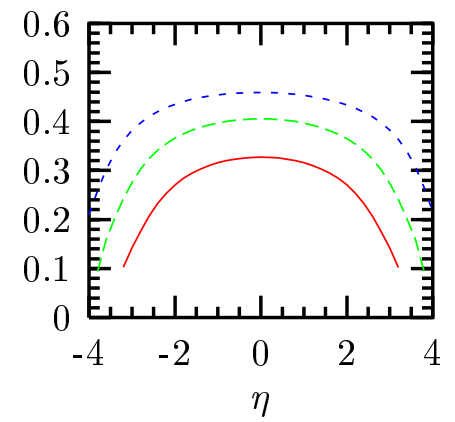

$\mathrm{RR}$

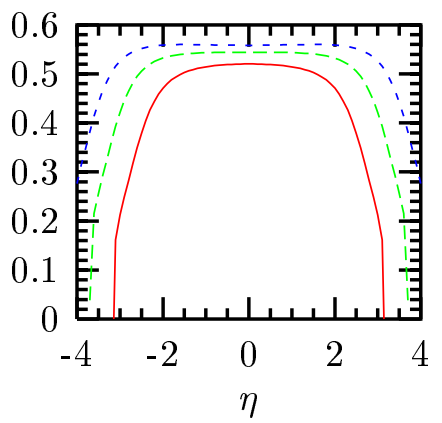

$\mathrm{DD}+\mathrm{DR}+\mathrm{RR}$

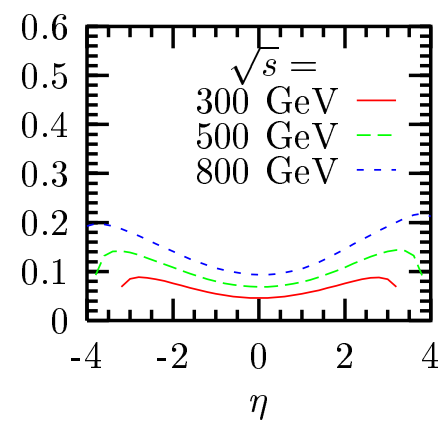

$\mathrm{DD}+\mathrm{DR}+\mathrm{RR}$

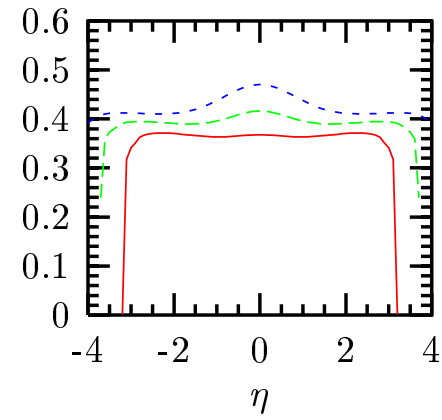


DR

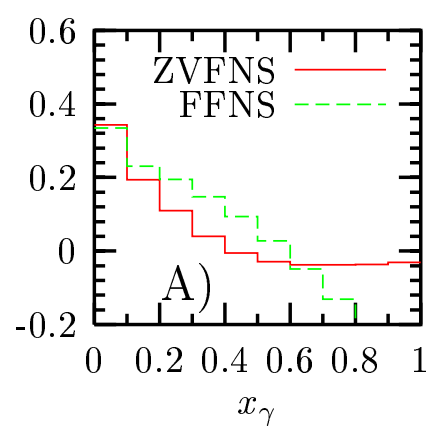

DR

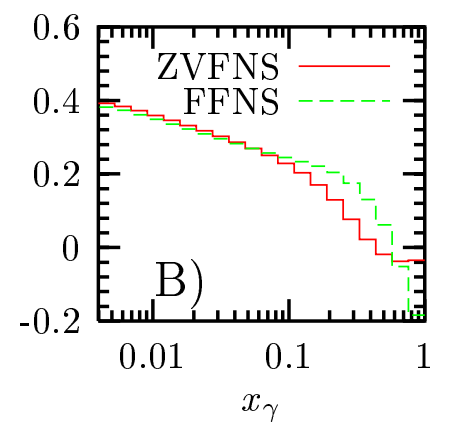

RR

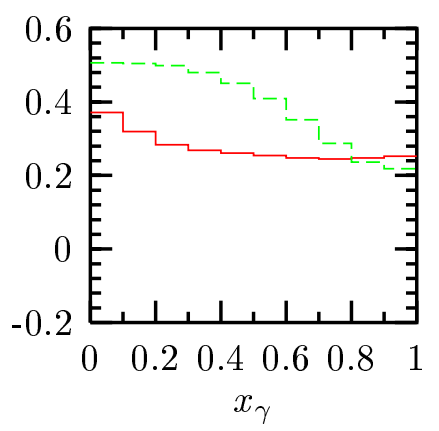

$\mathrm{RR}$

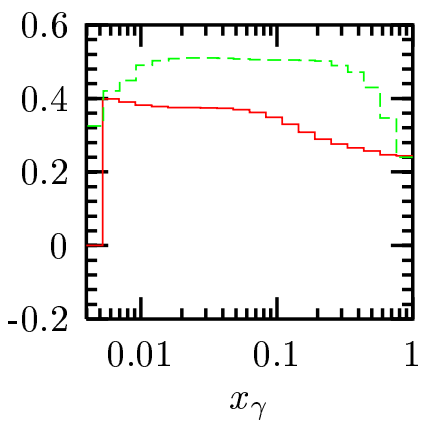

$\mathrm{DD}+\mathrm{DR}+\mathrm{RR}$

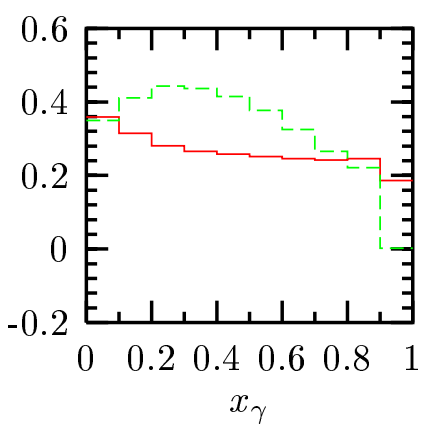

$\mathrm{DD}+\mathrm{DR}+\mathrm{RR}$

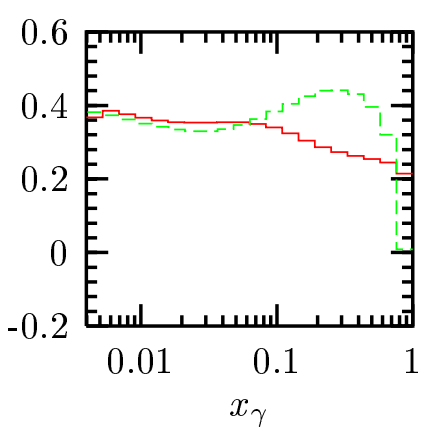

